\title{
Massive stars and their interstellar environment in the Magellanic Clouds
}

\author{
Michael A. Dopita ${ }^{1}$, Sungeun Kim ${ }^{1,4}$, M. Sally Oey ${ }^{2}$, \\ and Tatyana M. Lozinskaya ${ }^{3}$ \\ ${ }^{1}$ Mt. Stromlo $\&$ Siding Spring Observatory, \\ Research School of Astronomy \& Astrophysics, \\ Institute of Advanced Studies, Australian National University, \\ Australia, \\ ${ }^{2}$ Space Telescope Science Institute, Baltimore, MD, USA \\ ${ }^{3}$ Sternberg State Astronomical Institute, Moscow, Russia
}

\begin{abstract}
The winds of massive stars play a major role in setting up and controlling both the phase structure of the interstellar medium in galaxies and the vertical distribution of gas in galactic disks. In turn, these processes provide feedback into control of the star formation rate. Thanks to their proximity, these processes can be studied in detail in the Magellanic Clouds. Here we describe the results of a deep emission-line image survey of the ring nebula population, and a global high-resolution $\mathrm{H}$ I and $\mathrm{H}$ II survey of the Large Magellanic Cloud. These data provide a new insight into the process of feedback between the disk and the halo gas in disk galaxies in general, and the LMC in particular.
\end{abstract}

\section{Introduction}

The Magellanic Clouds present us with a unique opportunity to investigate the interaction of massive stars on their interstellar environment. First, the Magellanic Cloud stars are at a common distance and are close enough that individual stars and their stellar ejecta can be studied in detail. Second, the Large Magellanic Cloud is inclined at only $33^{\circ}$ to the line-of-sight, so that the 3-D structure of mass-loss bubbles can be mapped without problems of confusion, and third, the reddening is low in virtually all fields. In this paper we will show what has been learnt through recent surveys in $\mathrm{HI}$ and $\mathrm{H}$ II and X-rays about the way in which the energy released by the formation of massive stars generates a selfregulating feed back to maintain the phase structure of the interstellar medium in the Large Magellanic Cloud.

\section{Classification of the shell structures}

The Magellanic Clouds show shell structures ranging in size from individual stellar ejecta remnants which may be as small as one parsec across up to supergiant structures powered by several OB star clusters which are over one kiloparsec across. How should we classify these?

Clearly, the bubbles formed by individual early-type stars are easy to deal with; they fall into the classes which are broadly separated into age classes. The

\footnotetext{
${ }^{4}$ Present address: Steward Observatory, University of Arizona, Tucson, AZ, USA
} 
youngest are the mass-loss bubbles generated by the fast wind from the OB star. As the star transits towards the cool supergiant phase, and later, we have stellar ejecta shells being formed. As the star moves back towards the blue, these can be shocked, compressed and fragmented by the born-again fast wind. Finally in the Wolf-Rayet phase, a ring nebula can be formed, either as a result of passage of a stellar-wind powered shock through the stellar ejecta, or with the interstellar medium beyond the boundary of the OB star bubble. Of course, during any of the last three phases, the star may suddenly explode as a supernova and form a supernova remnant.

The larger bubbles formed by the collective effects of an OB association, or by triggered cascades of $\mathrm{OB}$ star clusters are rather more tricky to classify. Here we modify somewhat the nomenclature introduced by Meaburn (1980). Let us first define $\mathrm{HI}$ or $\mathrm{H}$ II giant shells as regions having a radius less than the scale height of the $\mathrm{HI}$ gas, which are therefore confined within the main $\mathrm{HI}$ layer in the LMC. In larger regions than this, the shape of the bubble will be very strongly modified by the density gradient, being flattened on the side nearest the galactic plane, and extended out of the plane.

Supergiant shells would then be those regions whose extent above the plane is so much larger than the $\mathrm{H}$ I scale height that the hot gas produced within the shell has broken out of the plane and is either draining out or has drained out its hot X-ray emitting gas into the hot halo gas around the LMC. Such regions form 'galactic chimneys' perpendicular to the disk plane. When it is drained of the overpressure which is driving the expansion of the $\mathrm{H}$ I shell, it has reached a momentum-conserving phase, and its expansion velocity in the galactic plane will reduce as more and more disk gas is swept up into the expanding $\mathrm{H}$ I shell. Unless it is powered by secondary star formation around its periphery, it will lose its identity either when the expansion velocity falls below the random turbulent velocity of the disk gas, or else when the differential velocity shear due to rotation becomes larger than the expansion velocity.

This provides a simple and physically meaningful distinction between the various shell classes. Let us now attempt to quantify this distinction, using the dynamical parameters we have determined for the H I disk in the LMC. For an isothermal disk, the scale height, $z$ is related to the velocity dispersion at the mid-plane, $v$, and the surface density of matter, $\mu$, as $z=\left\langle v^{2}\right\rangle / \pi G \mu$. In the van der Kruit \& Searle (1981a,b; 1982) disk model, the solution to the Poisson equation gives a vertical density profile $\rho(h)$ of the form $\rho(h)=\rho(0) \operatorname{sech}^{2}(h / z)$. At large height, this distribution is indistinguishable from an exponential. However, in a real galaxy the matter is not isothermal, since we have both gaseous and stellar components which, in general, have quite different velocity dispersions. Since the velocity dispersion of the gas component, $v_{g}$, is much smaller than the stellar component, $v_{*}$, the scale height of the gaseous component, $z_{g}$, is also much less than for the stars, $z_{*}$. In the particular case that the disk matter has an exponential distribution, and where the gas can be regarded as a sub-population in the same potential, but with different scale height, then the mid-plane velocity dispersion of the gas is given (exactly) by (Dopita \& Ryder 1994) as $v_{g}^{2}=2 \pi G \mu z_{g}^{2} /\left(z_{g}+z_{*}\right)$. Such an approximation is likely to be a reasonable approximation to the case of the LMC, provided that magnetic pressure support can be neglected. 
The $\mathrm{H}$ I rotation curve derived from the high-resolution maps by Kim et al. (1998a) implies a total disk mass of the LMC of $2.5 \times 10^{9} \mathrm{M}_{\odot}$ within a $4 \mathrm{kpc}$ radius. This covers most of the region in which bubbles and superbubbles are found, and which the rotation velocity is steeply rising. The mean (total) surface density of the disk is $48 \mathrm{M}_{\odot} \mathrm{pc}^{-2}\left(0.01 \mathrm{~g} \mathrm{~cm}^{-2}\right)$ and the RMS vertical velocity dispersion of the $\mathrm{HI}$ layer is $8 \mathrm{~km} \mathrm{~s}^{-1}$. On the other hand, the mean vertical velocity dispersion of the stars has been measured using the radial velocities of planetary nebulae by Meatheringham et al. (1988) at $19 \mathrm{~km} \mathrm{~s}^{-1}$. Substituting in the equations these figures give the scale height of the stars $\left(z_{*} \simeq 550 \mathrm{pc}\right)$ and the scale-height of the $\mathrm{HI}_{\mathrm{I}}\left(z_{\mathrm{g}} \simeq 190 \mathrm{pc}\right)$. We therefore set the division between shells and supershells at $D \simeq 380 \mathrm{pc}$. With this definition, the nature of the shells, their energy requirements and the shell sizes are listed in Table 1.

Table 1. Classification of shell structures

\begin{tabular}{|c|c|c|c|c|}
\hline central stars & shell type & $d E / d t\left(\operatorname{erg~s}^{-1}\right)$ & $E(\mathrm{erg})$ & $R(\mathrm{pc})$ \\
\hline individual OB/WR & bubble/ring nebula & $10^{36-37}$ & $10^{50-51}$ & $1-30$ \\
\hline OB association & giant shell & $10^{37-39}$ & $10^{51-53}$ & $50-180$ \\
\hline multiple OB assns. & supergiant shell & $>10^{38}$ & $>10^{53}$ & $>180$ \\
\hline
\end{tabular}

\section{Shells around Wolf-Rayet stars}

The population of the Wolf-Rayet stars has been very completely catalogued (Breysacher 1981, these Proceedings). In order to understand the nature and size distribution of the mass-loss bubbles about these, Dopita et al. (1994) made a deep $\mathrm{H} \alpha$ and [OIII] survey using the $A N U 2.3 \mathrm{~m}$ telescope. A number of different types are shown in Figure 1. Ring nebulae such as that associated with $\mathrm{Br} 10$ are mass-loss outflows interacting directly with the ISM in their vicinity. This particular object shows a fine development of the Rayleigh-Taylor instability, showing that the shell has recently been accelerated, and it also displays a global asymmetry caused by the gradient in the density of the local ISM. $\mathrm{Br} 48$, on the other hand shows the interaction of the WR wind on the $\mathrm{H}$ II region produced by the central star itself. $\mathrm{Br} 13$ and $\mathrm{Br} 31$ are examples of rings of stellar ejecta which have been thrown off in the red supergiant phase, and subsequently shocked by the born-again fast wind in the WR phase. Many of these WR stars lie in clusters which have themselves blown a giant shell $\mathrm{HII}$ region.

Stellar ejecta shells have also been seen about some cooler supergiant stars. Examples include Sk-69271 (B2) and Sk-69279 (O9f) (Weis et al. 1997).

\section{The H I shells}

The H I survey of the LMC has been presented by Kim et al. (1999). In brief, the LMC was divided into 12 regions, each one containing 112 pointing centers. The length of the integration at each pointing center was $15 \mathrm{~s}$, repeated approximately 

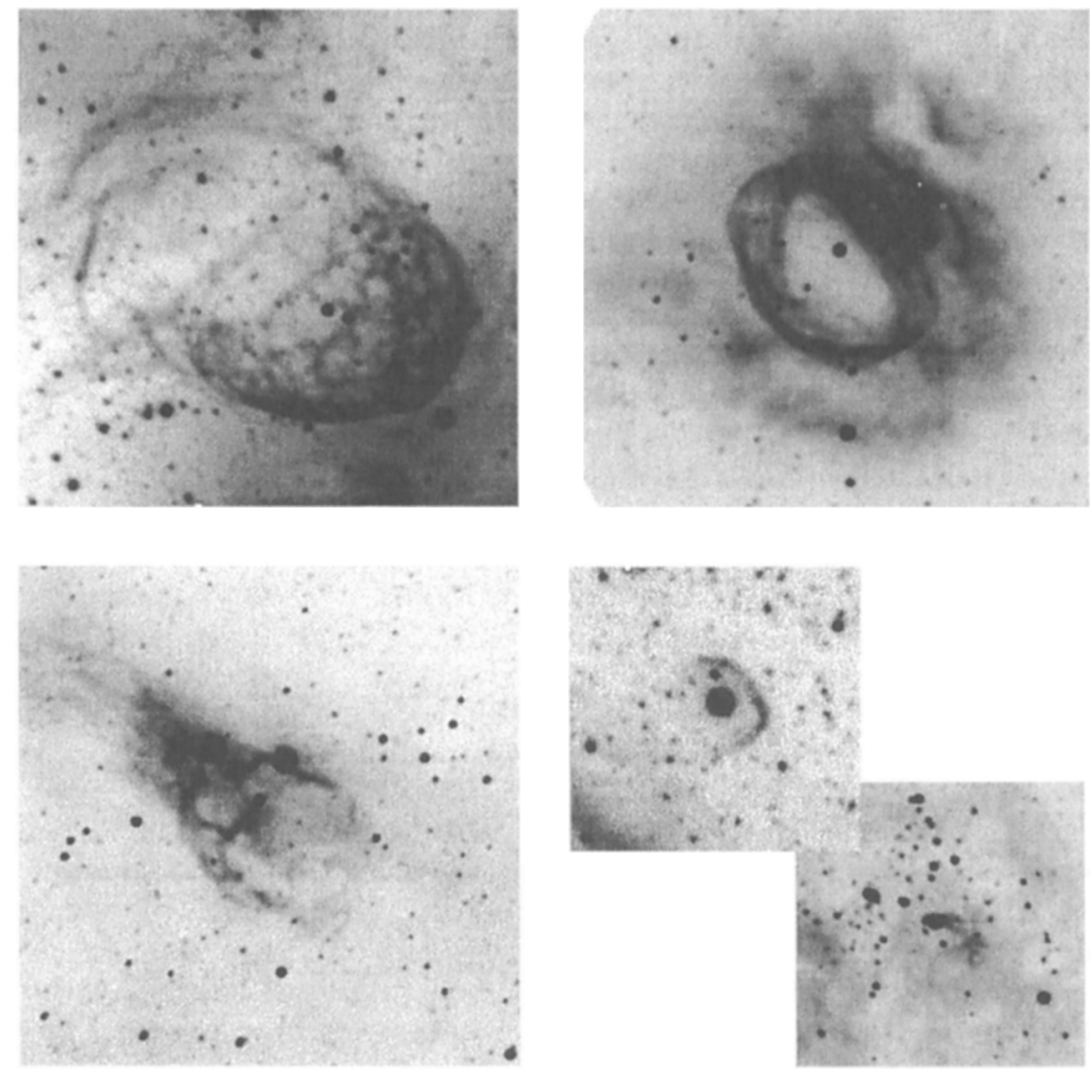

Figure 1. A selection of ring nebulae around WR stars in the LMC. From top left: $\mathrm{Br} 10, \mathrm{Br} 48, \mathrm{Br} 52, \mathrm{Br} 13$ and $\mathrm{Br} 31$. All are on the same scale, the large images covering $\sim 50 \mathrm{pc}$ at the distance of the LMC.

29 times during the course of a typical observing day of $15 \mathrm{~h}$. The pixel size is $20^{\prime \prime}$, the data were smoothed to a Gaussian beamwidth of FWHM $=1^{\prime} \times 1^{\prime}$ and the final size of the LMC data cube is $1998 \times 2230 \times 120$, corresponding to $11.1 \times 12.4^{\circ}$ on the sky with an RMS-noise of $9 \mathrm{~K}$. These maps were surveyed to find both giant and supergiant shells by searching for expanding elliptical regions in the channel maps. The global $\mathrm{H}$ I map was also compared with an $\mathrm{H} \alpha$ image taken with a focal reducing camera and covering the same region of sky as our $\mathrm{HI}$ survey (Kim et al. 1999). The spatial resolution was also quite similar, each pixel corresponding to $20^{\prime \prime} 63$ on the sky and covering a total field size of $11^{\circ} .7$.

The expansion velocity of the $\mathrm{H}$ I shells show a very clear correlation with the radius. For the giant shells, the expansion velocity increases from about $15 \mathrm{~km} \mathrm{~s}^{-1}$ for the smallest up to about $20-35 \mathrm{~km} \mathrm{~s}^{-1}$ showing that these are being accelerated by the energy input from the stars within them. The trend of velocity with radius is not an evolutionary one, since it is quite unlike that predicted by the standard Weaver et al. (1977) theory. In fact, the relationship 
arises from the intrinsic differences in mechanical energy input and ISM density in the giant shells of different sizes.

On the other hand, the supergiant shells show no trend with radius. The break in the expansion velocity versus radius relation occurs at a boundary between the two shell classes at $D=380 \mathrm{pc}$, and is consistent with the supergiant shells having exceeded their blowout diameter which we had estimated on the basis of the scale height of the matter in the disk of the LMC.

A high proportion of the giant and supergiant $\mathrm{H}$ I shells are colliding with one another. Many of them are interlocking, especially near the 30 Dor complex, where very active star formation has been occuring simultaneously in many different centres. The fact that the H I velocity structure in both the LMC and the SMC is dominated by a series of interlocking and interacting bubbles shows graphically that these are providing a fundamental control of the phase structure of the interstellar medium in these galaxies. This provides addition support to the model advocated by Oey \& Clarke (1997), who assumed that shell expansion is initially adiabatic, but stalls when the pressure in the bubble becomes comparable with the background pressure in the ISM. For a constant birthrate and a mechanical luminosity function $\phi(L) \propto L^{-\beta}$, this gives a predicted size distribution $N(R) \propto R^{1-2 \beta}$. The slopes predicted by this theory, using the observed luminosity distribution of the $\mathrm{H}$ II regions are in excellent accord with those derived directly from observation for both the SMC (Staveley-Smith et al. 1997) and the LMC (Kim et al. 1999).

\section{Relationship between the HI and HII shells}

Where $\mathrm{H}$ I shells are associated with $\mathrm{H}$ II regions, the $\mathrm{H}$ I structure is systematically larger than the $\mathrm{H}$ II structure in projection. This is to be expected since the $\mathrm{H}$ II region is confined in a thin layer between the ionisation front at the inner boundary of the $\mathrm{H}$ I shell and the hot shocked stellar wind material ejected by the central stars. In addition, we find that compact $\mathrm{H}$ II regions are preferentially located in, or on the boundaries of, dense $\mathrm{H}$ I filaments, as would be expected since star formation will naturally take place in the dense regions.

In general, however, there is only a weak correlation between the $\mathrm{H}$ I shells and the ionised gas traced out by the $\mathrm{H}$ II regions and $\mathrm{H}$ II shells. This is shown graphically in Figure 2, where the $\mathrm{HI}$ is shown in white tones, and the $\mathrm{HII}$ as dark tones. Since we believe that massive star formation is the ultimate causal factor of the $\mathrm{H}$ I shells and supershells, this shows that the either the lifetime of the $\mathrm{H}$ I shells is longer than the lifetime of the OB stars which input the energy required to drive the expansion of the $\mathrm{H}$ I shells, or that the pressure falls in the ionised regions sufficiently to lower the emission measure of the $\mathrm{H}$ II region below the threshold of observability. In fact both may be happening together, and indeed at low values of the emission measure, a diffuse ionised material is seen to pervade the whole galaxy (Kennicutt et al. 1995), much of which is associated with faint supergiant shells.

\section{Self-propagating star-formation}

It is clear that a number of the smaller shells have been formed on the rims of supergiant shells. This is clear observational support for a model of selfpropagating star-formation, where gravitational instabilities in the swept-up ma- 


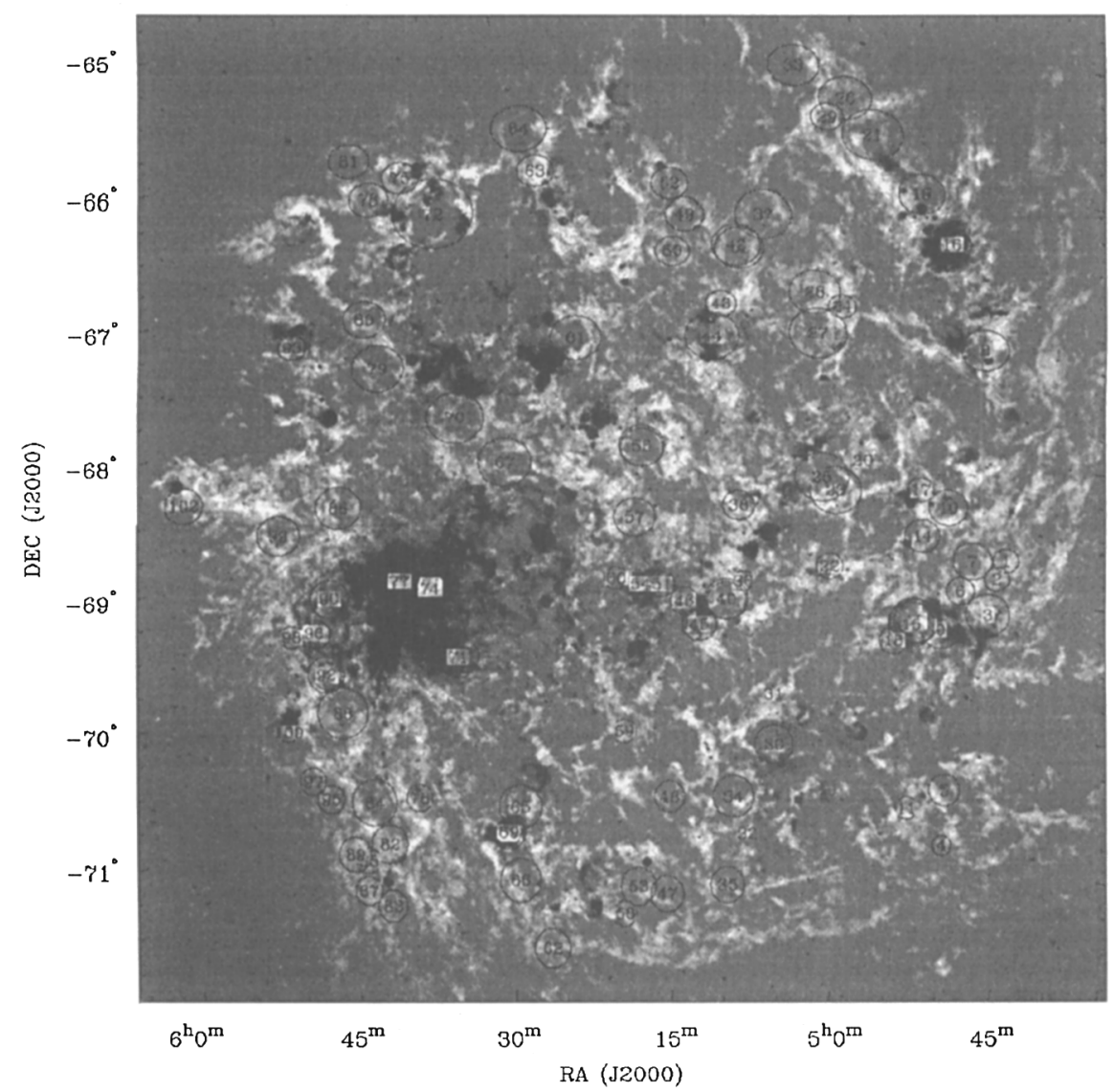

Figure 2. A comparison of the $\mathrm{H}_{\mathrm{I}}$ and $\mathrm{H}$ II in the LMC. The $\mathrm{H}$ I map is here shown in pale tones, and the $\mathrm{H}$ II regions in dark tones. Giant $\mathrm{H}$ I shells that have been identified from the channel maps are marked. This image emphasises the rather loose correlation between $\mathrm{H}$ I bubbles and the regions of active star formation as mapped by the HII, but shows how regions of star formation are concentrated in dense filaments of $\mathrm{HI}$.

terial of the supergiant shell caused fragmentation and a new round of star formation (Dopita et al. 1985; McCray \& Kafatos 1987; Elmegreen 1998; Efremov \& Elmegreen 1998). The best example of such self-propagating star formation in a supergiant shell is the LMC 4/Constellation III complex. This region in the northern part of the LMC, nearly $1.4 \mathrm{kpc}$ across, seems to have been initially triggered some $\sim 30 \mathrm{Myr}$ ago. This age is based on the existence of a central cluster of A-type supergiant stars (Efremov \& Elmegreen 1998), and this region seems to have passed through at least two episodes of triggered star-formation up to the present day, which first led to the formation of a great arc of stars (the "quadrant"), formed $\sim 10-18 \mathrm{Myr}$ ago, and finally to the set of $\mathrm{H}$ II regions 
and $\mathrm{H}$ I giant shells and supernova remnants (with ages of $\sim 1-10 \mathrm{Myr}$ ) which are currently located on the inner side of the $\mathrm{H}$ I supergiant ring.

Although the LMC4/Constellation III region is the best-known example, the 30 Dor region itself is a fine example of the phenomenon. The associated supergiant shell LMC-2, located just to the east of 30 Dor has been studied by Wang \& Helfand (1991) and Caulet \& Newell (1996).

Self-propagating star-formation is a phenomenon which extends down to the scale of giant $\mathrm{H}$ II shells, provided they occur in a dense enough region and provided that the local star-formation rate has been high enough. Examples include the DEM 34 (N 11) region (Walborn \& Parker 1992; Rosado et al. 1996) and the DEM 192/N 51D region (Oey \& Smedley 1998). A particularly fine example of a giant shell displaying self-propagating star formation is provided by the complex N 44 (Oey \& Massey 1995), recently studied by Kim et al. (1998b). This region contains three $\mathrm{OB}$ associations, and a number of single-star $\mathrm{H}$ II regions around the rim. The central association LH 47 is clearly older, since it contains a number of red supergiants. However, a WR star, $\mathrm{Br} 25$, is located on the SW boundary, and this is blowing a prominent ring nebula, N 44C. There is also a supernova remnant nearby. N 44 is filled with diffuse X-ray emission from hot plasma, which has already blown a pair of prominent $\mathrm{H}$ I shells. Both the $\mathrm{HI}$ and the $\mathrm{HI}$ regions are expanding at velocities between 30 and $60 \mathrm{~km} \mathrm{~s}^{-1}$. Nontheless, even N 44 shows the longstanding problem (Oey \& Massey 1995) that the apparent kinetic energy of the expansion is less than is expected by application of the Weaver et al. (1977) theory of pressure-driven bubbles. The probable solution to this is that a great deal of energy is stored in the motions of the molecular component which we cannot measure, since we have no molecular species we can use to trace the $\mathrm{H}_{2}$. (In Magellanic systems, the $\mathrm{CO}$ cannot be used, since $\mathrm{CO}$ is photo-dissociated, leading to an extremely low $\mathrm{CO}$ to $\mathrm{H}_{2}$ ratio).

Acknowledgments. The author wishes to acknowledge travel and publication support under a Major Grant from the International Science and Technology Division of the (Australian) Department of Industry, Science and Technology.

\section{References}

Breysacher, J. 1981, A\&AS 43, 203

Caulet, A., Newell, R. 1996, ApJ 465, 205

Dopita, M.A., Bell, J.F., Chu Y-H, Lozinskaya, T.A. 1994, ApJS 93, 544

Dopita, M.A., Mathewson, D.S., Ford, V.L. 1985, ApJ 297, 599

Dopita, M.A., Ryder, S.D. 1994, ApJ 430, 163

Efremov, Y.N., Elmegreen, B.G. 1998, MNRAS 299, 643

Elmegreen, B.G. 1998, in: C.E. Woodward, H.A. Thronson \& M. Shull (eds.), Origins of Galaxies, Stars, Planets and Life, ASP-CS 148, 149

Kim, S., Staveleley-Smith, L., Dopita, M.A., Freeman, K.C., Sault, R.J., Kesteven, M.J., McConnell, D. 1998a, ApJ 503, 674

Kim, Chu, Stavelely-Smith \& Smith 1998b, ApJ 503, 729

Kim, S. et al. 1999, ApJ in press

Kennicutt, R.C., Bresolin, F., Bomans, D.J., Bothun, G.D., Thompson, I.B. 1995, AJ 109,594 
McCray, R., Kafatos, M. 1987, ApJ 317, 190

Meaburn, J. 1980, MNRAS 289, 570

Meatheringham, S.J., Dopita, M.A., Ford, H.C., Webster, B.L. 1988, ApJ 327, 651

Oey, M.S., Clarke, C.J. 1997, MNRAS 289, 570

Oey, M.S., Massey, P. 1995, ApJ 452, 210

Oey, M.S., Smedley, S.A. 1998, AJ 116, 1263

Rosado, M., Laval, A., Le Coarer, E., Georgelin, Y.P., et al. 1996, A\&A 308, 588

van der Kruit, P.C., Searle L. 1981a, A\&A 95, 105

van der Kruit, P.C., Searle L. 1981b, A\&A 95, 116

van der Kruit, P.C., Searle L. 1982, A\&A 110, 61

Wang, Q., Helfand, D.J. 1991, ApJ 379, 327

Walborn, N.R., Parker, J.W. 1992, ApJ 399, L87

Weis, K., Chu, Y.-H., Duschl, W.J., Bomans, D.J. 1997, A\&A 325, 1157

\section{Discussion}

Marston: I didn't catch the resolution of your H I maps. Is it sufficient to indicate the atomic hydrogen content of nebulae associated directly with Wolf-Rayet stars?

Dopita: One arcminute, so in general, the answer to your question is no.

Dyson: In your spectacular photos of MC nebulae you suggested a RT-instability for an object when a shell appeared to be moving up a density gradient. This is the wrong direction for RT instability, since the shell needs to accelerate for RT instability.

Dopita: Yes, if it is decelerating, the RT-instability will not occur. On the other hand, the increase in mechanical energy-flux into the nebula may well have been sufficient to have accelerated the shell recently.

Terlevich: Is it possible at this stage to estimate the total H I mass and gas density of bubbles and loops as a fraction of the $\mathrm{H}$ I mass in high column-density regions? In other words, where is most of the $\mathrm{HI}$ in the LMC, in the loops or in the high column-density concentrations?

Dopita: Until we have the zero-spaced map to give us the true intensity map, this is not possible. I should emphasise that the $\mathrm{H}$ I map should be regarded as the radio-equivalent of an 'unsharp masked' image, and it has no 'D.C.' component.

Heckman: You have argued that the $\mathrm{H}$ I supershells without associated $\mathrm{H} \alpha$ emission are older systems, in which O-type stars are no longer present in the interior. Is this confirmed by the dynamical age-estimates for the expanding $\mathrm{H}$ I supershells?

Dopita: Yes.

Melnick: Do you confirm the result of the previous LMC H I map by Luks \& Rohlfs $(1992$, A\&A 263, 41) that the 30 Dor region is out-of the disk of the LMC, either up or down, by $\sim 400 \mathrm{pc}$ ?

Dopita: We find no evidence for a global distortion of the disk-plane, the 'spider diagram' is quite symmetric. However, we do find evidence for a ram-pressure compression and a tidal tail on the leading ( 30 Dor) edge of the LMC. This may well play a role in triggering the 30 Dor star-formation event. 